\title{
Nipple Pain, Damage, and Vasospasm in the First 8 Weeks Postpartum
}

\author{
Miranda L. Buck, Lisa H. Amir, Meabh Cullinane, \\ and Susan M. Donath ${ }^{2,3}$ for the CASTLE Study Team
}

\begin{abstract}
Background: Nipple pain and damage are common in the early postpartum period and are associated with early cessation of breastfeeding and comorbidities such as depression, anxiety, and mastitis. The incidence of nipple vasospasm has not been reported previously. This article describes nipple pain and damage prospectively in first-time mothers and explores the relationship between method of birth and nipple pain and/or damage.

Subjects and Methods: A prospective cohort of 360 primiparous women was recruited in Melbourne, Australia, in the interval 2009-2011, and after birth participants were followed up six times. The women completed a questionnaire about breastfeeding practices and problems at each time point. Pain scores were graphically represented using spaghetti plots to display each woman's experience of pain over the 8 weeks of the study.

Results: After birth, before they were discharged home from hospital, 79\% (250/317) of the women in this study reported nipple pain. Over the 8 weeks of the study $58 \%$ (198/336) of women reported nipple damage, and $23 \%$ $(73 / 323)$ reported vasospasm. At 8 weeks postpartum $8 \%(27 / 340)$ of women continued to report nipple damage, and 20\% (68/340) were still experiencing nipple pain. Ninety-four percent (320/340) of the women were breastfeeding at the end of the study, and there was no correlation between method of birth and nipple pain and/or damage.

Conclusions: Nipple pain is a common problem for new mothers in Australia and often persists for several weeks. Further studies are needed to establish the most effective means of preventing and treating breastfeeding problems in the postnatal period.
\end{abstract}

\section{Introduction}

$\mathbf{N}$ EW BREASTFEEDING MOTHERS are commonly advised that nipple tenderness should subside after the first week and that ongoing nipple pain is not normal. This picture of short-term discomfort is at odds with the reported experiences of mothers; for example, McCann et al. ${ }^{1}$ found that $38 \%$ of breastfeeding women interviewed at 1 month postpartum were experiencing persistent sore nipples. The phenomenological study of Williamson et al. ${ }^{2}$ described the surprise and confusion felt by women at the intensity and duration of the pain, and how they used terms such as "extremely, excruciatingly painful" and "horrific" to describe their experience of breastfeeding.

Nipple pain is second only to perceived low supply as the reason given by women for why they cease breastfeeding before they had planned to do so and is the most common reason for women to abandon breastfeeding before leaving the hospital. ${ }^{3-5}$ It may also lead to early supplementation with formula in the hospital, which is associated with reduced rates of breastfeeding at 6 months. ${ }^{6}$ Some women decide not to breastfeed altogether because they fear how painful it might be. Pain during breastfeeding is associated with depression, stress, sleep disturbances, and mastitis. ${ }^{7-10}$ Watkins et al., ${ }^{11}$ in their longitudinal cohort study of 2,586 women, found a twofold increase in the rate of depression at 2 months postpartum in women who had experienced severe pain on the first day of breastfeeding. Amir et al. ${ }^{7}$ demonstrated a correlation between breastfeeding pain and maternal distress and showed that depression scores normalized as breastfeeding difficulties were resolved. Although nipple pain and damage are significant problems leading to early cessation of

\footnotetext{
${ }^{1}$ Mother and Child Health Research, La Trobe University, Melbourne, Victoria, Australia.

${ }^{2}$ Department of Paediatrics, University of Melbourne, Melbourne, Victoria, Australia.

${ }^{3}$ Murdoch Childrens Research Institute, Melbourne, Victoria, Australia.

The CASTLE Study Team comprises Lisa H. Amir, Susan M. Donath, Suzanne M. Garland, Sepehr N. Tabrizi, Catherine M. Bennett, Meabh Cullinane, and Matthew S. Payne.
} 
breastfeeding and maternal distress, detailed longitudinal studies of such complications are missing from the literature.

There are several causes of nipple pain, including trauma, such as from a poor latch or tongue-tie, infection, and vasospasm. ${ }^{12}$ McClellan et al. ${ }^{13}$ found that some babies exerted a higher vacuum than others, which was painful for their mothers. However, risk factors that may be associated with nipple pain have not specifically been identified. Cesarean section rates are increasing worldwide, and our clinical experience suggested that following cesarean section women experience more nipple damage, and therefore pain, than women giving birth vaginally. ${ }^{14}$ The study by Kearney et al. ${ }^{15}$ of 121 primiparous women found that $37 \%$ experienced breastfeeding pain but that breastfeeding problems did not vary by mode of delivery. Zanardo et al. ${ }^{16}$ differentiated between elective and emergency cesareans and demonstrated an association between elective cesarean delivery and early cessation of breastfeeding. In particular, they noted that elective cesarean was associated with not initiating breastfeeding while in the hospital, but they did not take into consideration the level of women's breastfeeding motivation. ${ }^{16}$ Suzuki et al. ${ }^{17}$ reported higher rates of exclusive breastfeeding after cesarean birth in one Tokyo hospital, but in unusually supportive circumstances. We have not identified any studies that have investigated if nipple pain and damage vary by mode of delivery.

Nipple vasospasm has been described in case studies of breastfeeding women as a reduced flow of blood through the capillaries caused by constriction in the peripheral circulation. ${ }^{18-22}$ It may be exacerbated by cold and a poor latch. The symptoms are blanching of the tissues, followed by reperfusion where a color change is noted from white to cyanosed to red. This intermittent ischemia may be acutely painful. It has been associated with Raynaud's phenomenon, the use of some medications during pregnancy, breast surgery, and autoimmune disease. ${ }^{23}$ Incidence of nipple vasospasm is currently unknown, but there have been reports of successful treatment with calcium and magnesium supplementation and with the calcium channel blocker nifedipine. ${ }^{18,24}$

This article reports breastfeeding women's nipple pain and damage, over the first 8 weeks postpartum, and explores the relationship between method of birth and nipple pain and damage. Data were collected as part of the CASTLE (Candida and Staphylococcus Transmission: Longitudinal Evaluation) study, a longitudinal descriptive study of breastfeeding mothers and their babies. ${ }^{25}$ The CASTLE study was designed to investigate the role of Staphylococcus aureus and Candida in breast pain and infection among lactating women, and the main results have been published elsewhere. ${ }^{26}$

\section{Subjects and Methods}

The main aims of the CASTLE study was to determine whether S. aureus or Candida albicans is the primary organism involved in breast thrush in lactating women, and further details are available in the published protocol. ${ }^{25}$ An additional component to the overall CASTLE study, MOAT, is also described in the study protocol. Most of the women recruited to CASTLE also completed MOAT, a written self-report questionnaire composed of the Profile of Mood States, the Depression, Anxiety and Stress Scale, and the Vulnerable Personality Scale. The findings from MOAT are currently being analyzed for publication.

\section{Setting}

Primiparous women were recruited from The Royal Women's Hospital, a public tertiary women's hospital in Melbourne, VIC, Australia, and Frances Perry House, a private hospital located on the same site. The Royal Women's Hospital has been accredited as Baby Friendly since 1995. Both sites encourage breastfeeding with postpartum rooming-in, and dedicated lactation support services are available on demand.

The participants were all made aware of the Australian Breastfeeding Association Mum-2-Mum 24-hour free telephone breastfeeding helpline, a national voluntary organization staffed by trained breastfeeding counselors. Public hospital patients were visited at home at least once by a domiciliary midwife, and all mothers were offered appointments by the universal Maternal and Child Health Nurse Service including a minimum of one home visit and then consultations at 2, 4, and 8 weeks. The Maternal and Child Health Service in Victoria also offers a 24-hour, 7 days a week, information and support telephone service. The Royal Women's Hospital Breastfeeding Support Service offers a comprehensive outpatient lactation support service to women who have given birth at The Royal Women's Hospital, and the lactation consultants are accredited to perform tongue-tie release. $^{27}$

\section{Study sample}

A prospective cohort of 360 primiparous women was recruited from November 2009 to June 2011. The eligibility criteria for the study were as follows: between 18 and 50 years of age; nulliparity; $\geq 36$ weeks pregnant at recruitment; singleton pregnancy; breastfeeding intention for at least 8 weeks postpartum; sufficient proficiency in English to complete written questionnaires and a telephone interview; and residing $\leq 16 \mathrm{~km}$ from Melbourne Central Business District. Criteria for exclusion were as follows: medical conditions that do not allow breastfeeding; breast reduction surgery; dermatitis on nipple during pregnancy; under care of the Women's Hospital Alcohol and Drug Service; or under care of the Mental Health Service or a social worker. These were all motivated women who intended to breastfeed for at least 8 weeks, and many of them were recruited at the hospital antenatal breastfeeding classes.

\section{Procedures}

As part of the overall CASTLE study, at recruitment, nasal, nipple (both breasts), and vaginal swabs were collected, and participants completed a short questionnaire. Following the birth, participants were followed up six times: in the hospital and then at home weekly until 4 weeks postpartum and by telephone at 8 weeks. At each time point participants completed a questionnaire about breastfeeding practices and problems, based on previous research. ${ }^{28}$ This article presents the results of the following questions:

- "In the last 48 hours have you been experiencing nipple pain/discomfort?

- "How would you describe your worst experience of nipple pain in the last 24 hours?" The response used a numeric scale from 0 to 10 , where 0 means "no pain at all" and 10 means "the worst pain possible." 
Table 1. Characteristics of Primiparous Women Recruited in Late Pregnancy

\begin{tabular}{lc}
\hline Maternal characteristics (n=346) & $\mathrm{n}(\%)$ \\
\hline Hospital & \\
$\quad$ Royal Women's Hospital (public) & $154(44.5)$ \\
$\quad$ Frances Perry House (private) & $192(55.5)$ \\
Age (years) [mean (SD, range) & $32.7(4.1,19-44)$ \\
Marital status & \\
$\quad$ Married & $229(66)$ \\
Unmarried, living with partner & $103(30)$ \\
$\quad$ Not living with partner & $2(1)$ \\
Separated/divorced & $1(0)$ \\
$\quad$ Single & $11(3)$ \\
Education level & \\
$\quad$ Tertiary degree or higher & $267(77)$ \\
Gestation at (weeks) [mean (SD, range)] & \\
$\quad$ Recruitment & $37(1.3,34-42)$ \\
$\quad$ Birth & $39(1.2,36-42)$ \\
Breastfeeding intention & $9.7(1-24)$ \\
$\quad$ (months) [mean (range)] & $156(45)$ \\
Cesarean birth & $168(49)$ \\
Baby's sex male & $320(94)$ \\
Any breastmilk feeding & \\
at 8 weeks postpartum $(n=340)$ & \\
\hline
\end{tabular}

- "Do you have nipple damage?" Response options included "No," "Yes, small graze/crack ( $<2 \mathrm{~mm}$ in length)," "Yes, moderate graze/crack (2-9 mm in length)," "Yes, severe graze/crack (10 mm or larger and/or yellow color present)," and "Not sure."

- "If your nipple is cracked, does the crack in your nipple open up more after you feed or express?" Response options included "No," "Yes, a little," "Yes, a lot," "Not sure," and "Not applicable."

- "Do you have nipple vasospasm (nipple blanches or goes white in the cold or during/after feeds)?" Response options included "No," "Yes, for less than 5 minutes," "Yes, for more than 5 minutes," "Yes, but not sure how long," and "Not sure."

This study was approved by the La Trobe University Human Ethics Committee (approval number 06-078), the $\mathrm{Hu}$ man Research Ethics Committee of the Royal Women's Hospital (approval number 06/41), the Human Research Ethics Committee of the University of Melbourne (approval number 1033949), and the Medical Advisory Committee at Frances Perry House.
Table 2. Mean and Median Pain Scores by Week

\begin{tabular}{lccc}
\hline Week & Mean (n) & Median & $\begin{array}{c}\text { Mean of women with } \\
\text { vasospasm for }>5 \text { minutes } \\
\text { at Week } 8(\mathrm{n})\end{array}$ \\
\hline Hospital & $2.8(312)$ & 2.0 & $2.5(14)$ \\
Week 1 & $3.4(336)$ & 3.0 & $4.5(17)$ \\
Week 2 & $2.4(336)$ & 2.0 & $2.7(16)$ \\
Week 3 & $2.3(326)$ & 2.0 & $3.2(17)$ \\
Week 4 & $1.6(323)$ & 0.0 & $3.5(15)$ \\
Week 8 & $0.8(339)$ & 0.0 & $1.6(17)$ \\
\hline
\end{tabular}

Pain scores include scores of zero; zero is no pain, and 10 is the worst pain possible.

\section{Analysis}

Statistical analysis was conducted using Stata version 12 software (StataCorp LP, College Station, TX). Descriptive data are presented in the tables. The nipple pain data are displayed as spaghetti plots, which enable trends, clusters, and outliers in the structure of the data to be explored. ${ }^{29}$ The spaghetti plots are graphical representations summarizing the longitudinal data; the trajectories track pain scores of each woman over time. We had previously estimated that a sample of 318 women would provide adequate power for the main hypothesis of the study. ${ }^{25}$ We considered $p$ values $<0.05$ as statistically significant. Levels of nipple pain in women who had a cesarean delivery were compared with those who had a vaginal birth using $\chi^{2}$ tests. To investigate the relationship between nipple vasospasm and nipple pain over the 8 weeks, linear regression was used.

\section{Results}

Of the 360 women recruited, 14 withdrew or were lost from the study after giving birth, leaving 346 (96\%) women available for data collection; 340 (94\%) women completed the study at 8 weeks postpartum. This cohort of first-time mothers was well educated, with $77 \%$ of them having achieved a tertiary degree or higher (Table 1). Recruitment commenced at 36 weeks of gestation, and gestational age at birth ranged from 36 to 42 weeks. The participants all intended to breastfeed for at least 8 weeks, and $65 \%$ of them intended to continue for 6 months or more (223/345). The overall cesarean rate was $45 \%$, and $56 \%$ gave birth in the private hospital.

In the first few days after birth, before they were discharged home from hospital, $79 \%(250 / 317)$ of the women reported nipple pain. The proportion of women experiencing pain

Table 3. Women Experiencing Nipple Pain and Damage by Mode of Birth

\begin{tabular}{lccccc}
\hline Nipple condition, mode of birth & Week $1(\mathrm{n}=336)$ & Week $2(\mathrm{n}=336)$ & Week $3(\mathrm{n}=326)$ & Week $4(\mathrm{n}=323)$ & Week $8(\mathrm{n}=340)$ \\
\hline Nipple pain & & & & & \\
$\quad$ Vaginal & $131(70)$ & $106(56)$ & $95(52)$ & $78(43)$ & $39(21)$ \\
$\quad$ Cesarean & $112(75)$ & $91(62)$ & $88(61)$ & $62(44)$ & $30(19)$ \\
$\quad$ Total & $243(72)$ & $197(59)$ & $183(56)$ & $140(43)$ & $69(20)$ \\
Nipple damage & & & & \\
$\quad$ Vaginal & $111(60)$ & $58(31)$ & $52(29)$ & $44(24)$ & $20(10)$ \\
$\quad$ Cesarean & $84(56)$ & $53(36)$ & $40(28)$ & $35(25)$ & $7(5)$ \\
$\quad$ Total & $195(58)$ & $111(33)$ & $92(28)$ & $79(24)$ & $27(8)$ \\
\hline
\end{tabular}

Data are number (\%). 


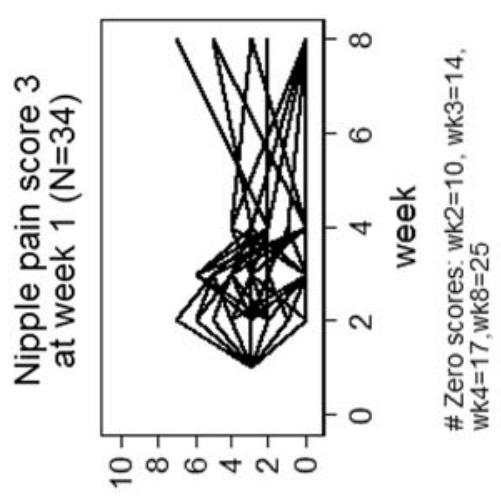

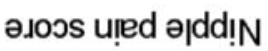

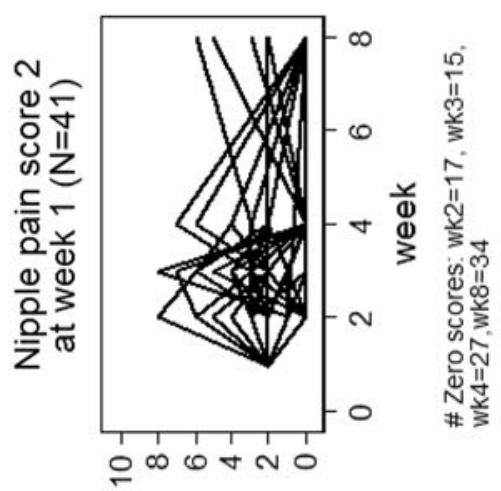

әرоэ u!̣ed әృdd!N

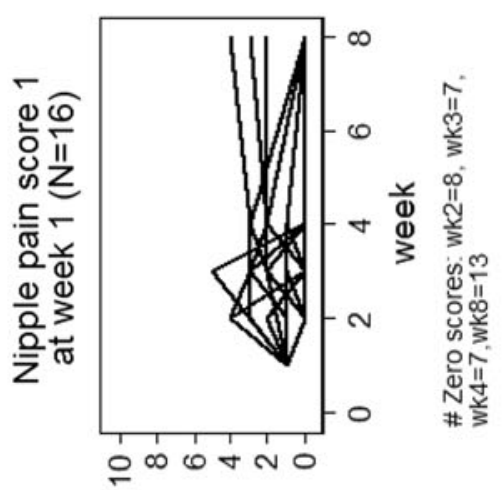

әرоэs u!̣ed ә|dd!n

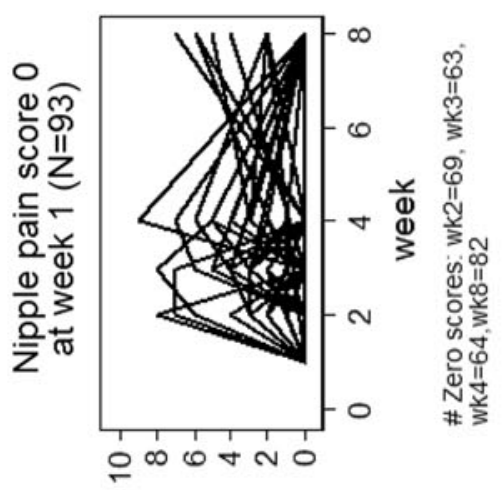

әıоэs u!̣ed әృdd!N

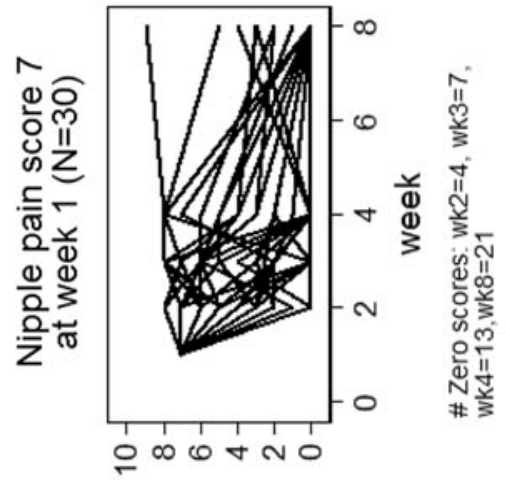

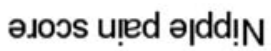

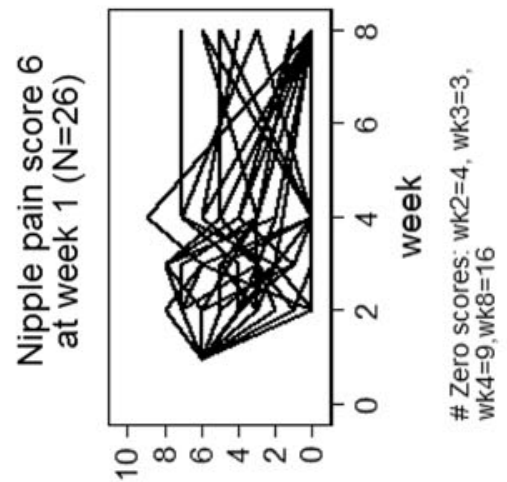

ə.০os uा̣ed ə|dd! N

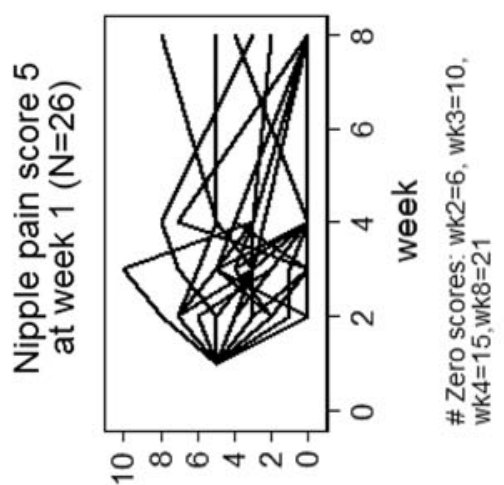

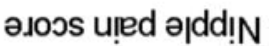

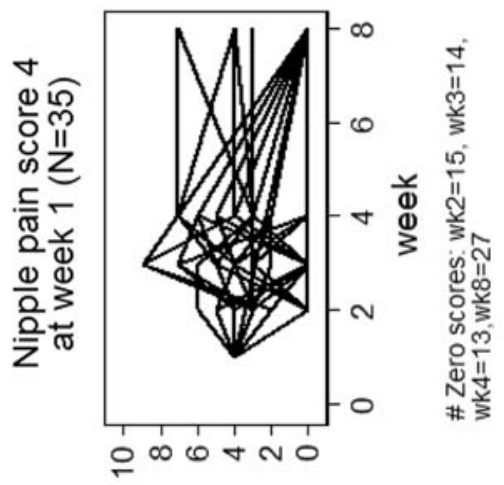

әرoวs uाed ә|dd!n

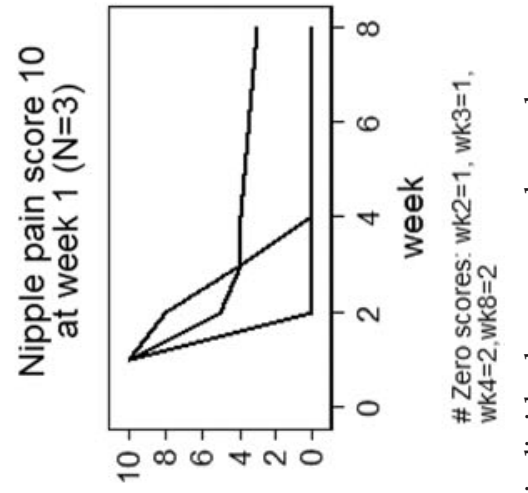

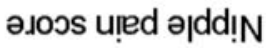

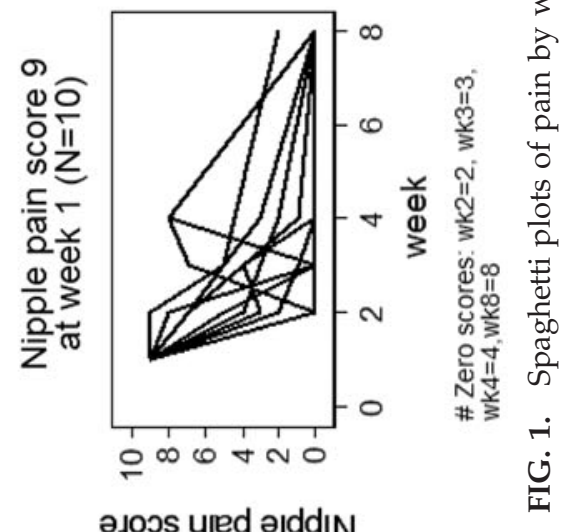

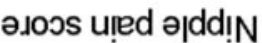

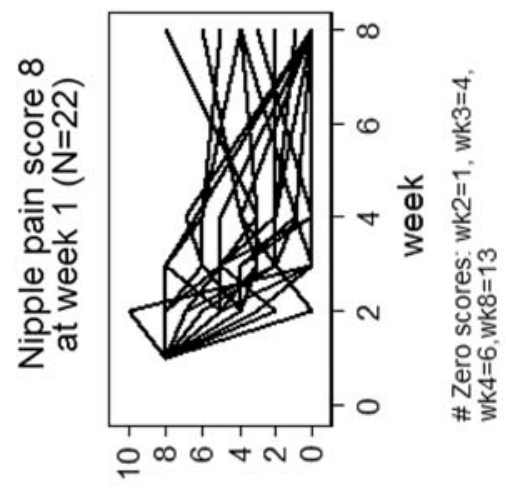

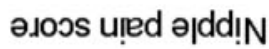


Table 4. Nipple Damage by Size of Crack

\begin{tabular}{|c|c|c|c|c|c|}
\hline & Week $1(\mathrm{n}=336)$ & Week $2(\mathrm{n}=336)$ & Week $3(\mathrm{n}=326)$ & Week $4(\mathrm{n}=323)$ & Week $8(\mathrm{n}=340)$ \\
\hline No crack & $133(40)$ & $213(63)$ & $224(69)$ & $234(72)$ & $314(91)$ \\
\hline Small $^{\mathrm{a}}$ & $134(40)$ & $70(21)$ & $60(18)$ & $54(17)$ & $15(4)$ \\
\hline Moderate $^{b}$ & $54(16)$ & $34(10)$ & $30(9)$ & $24(7)$ & $11(3)$ \\
\hline Severe ${ }^{c}$ & $7(2)$ & $7(2)$ & $2(1)$ & $1(1)$ & $1(1)$ \\
\hline Unsure & $8(2)$ & $12(4)$ & $10(3)$ & $10(3)$ & $3(1)$ \\
\hline
\end{tabular}

Data are number (\%).

${ }^{\mathrm{a}} \mathrm{Small}=<2 \mathrm{~mm}$ in length.

${ }^{\mathrm{b}}$ Moderate $=2-9 \mathrm{~mm}$ in length.

'Severe $=10 \mathrm{~mm}$ or larger and / or yellow color present.

gradually decreased over the study period, but the improvements in comfort were gradual, as shown in Table 2. More than half of the women (183/326) were still experiencing discomfort 3 weeks after giving birth (Table 3). By 8 weeks postpartum $20 \%$ of women continued to be in pain.

The spaghetti plots (Fig. 1) illustrate the individual pain scores of women during the first 8 weeks of breastfeeding. Experiencing little or no pain in the first week of breastfeeding did not predict a pain-free course. Of the 93 women who rated their pain as zero in the first week, $26 \%$ experienced pain during the second week, $32 \%$ reported pain at Week 4 , and $12 \%$ reported pain in Week 8 . There were 26 women who rated their pain as 6 out of 10 in their first week of breastfeeding. By their third week of breastfeeding $88 \%$ of them were still in pain, and 39\% remained in discomfort at the end of the study. Although nipple pain generally decreased in severity over the weeks and most women were free of pain by Week $8,35 \%(24 / 68)$ of the women who continued to experience nipple pain after 8 weeks of breastfeeding rated their pain as 5 or more out of 10 .

Small numbers of women reported nipple damage while in the hospital, but grazed and cracked nipples were an increasing problem in the first week of breastfeeding, with $58 \%$ of women experiencing some damage (Table 4). After 8 weeks of breastfeeding $8 \%(27 / 340)$ of women continued to report nipple damage, and 11 of those had moderate $(2-9 \mathrm{~mm})$ or severe cracks ( $10 \mathrm{~mm}$ or more) that opened up during feeds.

At all times in this study a small proportion of women reported that they experienced vasospasm. In the first week $14 \%(45 / 336)$ of women reported symptoms of vasospasm. At Week 4 23\% (72/323) of participants reported vasospasm: $16 \%(51 / 323)$ for less than 5 minutes, 3\% (10/323) for more than 5 minutes, and $4 \%(12 / 323)$ were unsure how long the vasospasm lasted. At Week 8 postpartum these figures remained stable, with $22 \%$ (77/340) of participants continuing to experience vasospasm. None of the women reported taking any treatment, such as nifedipine, during the study. The mean pain scores of the women with vasospasm were higher than those without symptoms of vasospasm (Table 2). When variations in pain score at each time point are taken into account, on average women with vasospasm had high pain scores higher than women with no vasospasm at 8 weeks $(p=0.02$, regression coefficient $=0.86,95 \%$ confidence interval $0.13,1.58$ ).

Table 3 displays nipple pain and damage according to mode of delivery. There was no correlation between nipple pain and mode of delivery.

Exclusive breastfeeding at the breast was achieved by $66 \%$ $(227 / 346)$ of women by the end of the study, and exclusive breastmilk feeding was achieved by $80 \%(277 / 346)$. At 8 weeks postpartum two babies were exclusively fed on expressed breastmilk. Formula supplementation to breastfeeding was being practiced with $13 \%$ of babies, and $6 \%(20 / 346)$ were fully formula fed. Overall, $94 \%(326 / 246)$ of babies in this study were receiving any breastmilk at 8 weeks of age. The women who had ceased breastfeeding were asked why, and one woman indicated that nipple trauma was one of the reasons she ceased breastfeeding, but none of the women said it was their main reason for discontinuing. The most common reason given for ceasing breastfeeding was that women felt there was not enough milk (50\% [9/18]). Other reasons each given by individual women included the following:

- "It was taking too long with feeding at the breast."

- "Had got used to bottle, wouldn't take breast"

- "I needed to gauge how much baby was getting."

- "Milk never came in."

- "Mother taking medication [eye drops]"

\section{Discussion}

Despite a high motivation to breastfeed, access to education, a Baby Friendly institution, and extensive postnatal support, $79 \%$ of new mothers in this study experienced nipple pain, and $58 \%$ had damaged nipples that were cracked or grazed. The proportion of women experiencing nipple pain is remarkably similar to that found in the 1950s in Philadelphia by Newton. ${ }^{30}$ This seems high, when compared with some studies such as that of Doherty et al., ${ }^{31}$ who described $11 \%$ of breastfeeding women in South Africa in their study suffering from any breast health problem, and $23 \%$ of Peruvian women, described by Strong, ${ }^{32}$ as reporting breastfeeding pain. This well-educated sample of women had access to a number of resources to support their breastfeeding intention, and yet their problems persisted for weeks.

Breastfeeding practices such as skin-to-skin contact at birth between mother and baby and initiating breastfeeding within the first hour of life improve breastfeeding outcomes, but evidence on the prevention of breastfeeding pain is less clear. ${ }^{33}$ Breastfeeding is a cultural practice, but in Australia it is a skill where "...social or embodied learning is now a rare phenomenon and so new ways of learning need to be found." 34 , p.163 Intervention studies have had mixed success with preventing breastfeeding problems through programs of antenatal education, and informal information available via the Internet about breastfeeding is often inaccurate, contradictory, and unhelpful. ${ }^{35,36}$ It has been argued that the fundamental problem is a mismatch between the cultural habits of Australian mothers and the primitive neonatal reflexes of babies. ${ }^{37}$ For example, Doucet et al. ${ }^{38}$ suggested that 
the secretions of Montgomery glands are an important cue for infants in latching, and it may be that some hygiene practices disrupt this pathway and may lead to less effective suckling. It may be that a combination of birthing, infant care, hygiene, and breastfeeding practices acts together to destabilize early establishment of comfortable and effective breastfeeding.

Nipple vasospasm has been described as underdiagnosed ${ }^{22}$ and has mainly been reported as case studies. ${ }^{18,21,23}$ Raynaud's phenomenon is clinically diagnosed based on signs and symptoms, ${ }^{39}$ and there are several variations of peripheral vasospasm described in the literature as related to Raynaud's phenomenon. Vibration-induced white finger syndrome, which can be graded using the Taylor-Pelmear scale, is a more common presentation in men and has a prevalence of $0-5 \%$ in warm climates and up to $80-100 \%$ in cold climates. ${ }^{40}$ In women peripheral vasospasm is associated with estrogen exposure, emotional stress, and a history of migraines. ${ }^{41}$ It seems likely that a combination of hormonal, emotional, and physiological changes associated with childbirth and the mechanical forces of the breastfeeding infant compound to produce the vascular symptoms observed in some breastfeeding women. A postal survey in the United Kingdom found that $14 \%$ experienced finger blanching, but only one-fifth of respondents had seen their doctor about it, which suggests that this phenomenon is common and not always troublesome in the general population. ${ }^{42}$

A fifth of the CASTLE participants experienced vasospasm, and for most of these women it was not a significant problem. Although their pain scores were generally higher than those women without vasospasm, none of them had commenced nifedipine by Week 8 . There are no published indications of prevalence of nipple vasospasm, so it is not possible to say if these figures are high or what may be found in any population of breastfeeding women. Raynaud's phenomenon is reported to affect $20 \%$ of women of childbearing age, so it may be that it is not a coincidence that $20 \%$ of the women in this study also reported similar symptoms. Anderson et al. ${ }^{19}$ cautioned that the symptoms of nipple vasospasm may be easily mistaken for those of nipple thrush, such as throbbing breast pain, and that a careful history must be taken to avoid incorrect prescription of antifungals. Nipple vasospasm may be effectively treated by applying warmth and nifedipine, and there are lay reports of calcium, magnesium, and fish oil supplements being helpful. ${ }^{18,19}$

Overall, $94 \%$ of babies in this study were receiving any breastmilk at 8 weeks of age, $81 \%$ were fully breastmilk fed, and $67 \%$ were fully breastfeeding. This is higher than the $57 \%$ of babies reported to be fully breastfed at 8 weeks in the 2010 Australian National Infant Feeding Survey. ${ }^{43}$ We found no difference in nipple pain or damage according to method of birth. This intentional sample of women who planned to breastfeed for at least 8 weeks is not representative of all women, and so the results are not comparable to those determined by researchers such as Ahluwalia et al., ${ }^{44}$ who found that women who delivered by cesarean section breastfed for an average of 20 weeks versus 45 weeks for normal vaginal births and that women who had an elective cesarean were more likely to not wish to breastfeed at all.

The main strength of this study is the level of detail that was collected prospectively from a large group of women. We present the pain scores of every woman, over time, which enables the patterns and outliers in the data to be visualized. Additionally, this study was performed in two excellent maternity facilities where the women were receiving a high standard of care, which is arguably as close to best practice in terms of supporting women to breastfeed, as we could have found in Australia.

There are some limitations to this study. Although the women were cared for by midwives and doctors who have had training in supporting women to breastfeed and had access to skilled support, their breastfeeding itself was not assessed by the researchers in this study. We report here the symptoms of breastfeeding problems, and although we can report that $94 \%$ of these women continued to breastfeed at 8 weeks postpartum, we cannot assess the cause of their pain or damage during this study. The women in this study were a fairly homogeneous sample of well-educated and motivated women, who all intended to breastfeed for at least 8 weeks and most for at least 6 months. This would not be comparable to either state or Australian populations, but it further emphasizes that despite their privilege this group experienced a significantly troubled time establishing breastfeeding.

\section{Conclusions}

Nipple pain is a common problem for new mothers in Australia and often persists for several weeks. Preventing breastfeeding problems from arising and rapidly addressing those problems that do present in the early postpartum period are essential to the provision of perinatal care, in order to optimize the health and well-being of mothers and babies. The most effective means of helping mothers to establish comfortablebreastfeeding and continue to breastfeed as long as they wish to has yet to be established, and it is research that is urgently needed.

\section{Acknowledgments}

This study received financial support from the National Health \& Medical Research Council (project grant 541907, equipment grant, Health Professional Training Fellowship [to L.H.A.]), Helen Mcpherson Smith Trust, Faculty Research Grant, Faculty of Health Sciences, La Trobe University. M.L.B. has a Faculty of Health Sciences, La Trobe University, Dean's Scholarship.

\section{Disclosure Statement}

No competing financial interests exist.

\section{References}

1. McCann MF, Baydar N, Williams RL. Breastfeeding attitudes and reported problems in a national sample of WIC participants. J Hum Lact 2007;23:314-324.

2. Williamson I, Leeming D, Lyttle $S$, et al. 'It should be the most natural thing in the world': Exploring first-time mothers' breastfeeding difficulties in the UK using audiodiaries and interviews. Matern Child Nutr 2012;8:434-447.

3. Li R, Fein SB, Chen J, et al. Why mothers stop breastfeeding: Mothers' self-reported reasons for stopping during the first year. Pediatrics 2008;122(Suppl 2):S69-S76.

4. Scott JA, Landers MCG, Hughes RM, et al. Psychosocial factors associated with the abandonment of breastfeeding prior to hospital discharge. J Hum Lact 2001;17:24-30.

5. Odom EC, Li R, Scanlon KS, et al. Reasons for earlier than desired cessation of breastfeeding. Pediatrics 2013;131:e726e732. 
6. Forster D, McLachlan H, Lumley J. Factors associated with continuing to feed any breast milk at six months postpartum in a group of Australian women. Int Breastfeed J 2006;1:18.

7. Amir LH, Dennerstein L, Garland SM, et al. Psychological aspects of nipple pain in lactating women. J Psychosom Obstet Gynaecol 1996;17:53-58.

8. McClellan HL, Hepworth AR, Garbin CP, et al. Nipple pain during breastfeeding with or without visible trauma. J Hum Lact 2012;28:511-521.

9. Amir LH, Forster D, Lumley J, et al. A descriptive study of mastitis in Australian breastfeeding women: Incidence and determinants. BMC Public Health 2007;7:62.

10. Annagür A, Annagür BB, Şahin A, et al. Is maternal depressive symptomatology effective on success of exclusive breastfeeding during postpartum 6 weeks? Breastfeed Med 2013;8:53-57.

11. Watkins S, Meltzer-Brody S, Zolnoun D, et al. Early breastfeeding experiences and postpartum depression. Obstet Gynecol 2011;118:214-221.

12. Eglash A, Montgomery A, Wood J. Breastfeeding. Disease- $A-$ Month 2008;54:343-411.

13. McClellan H, Geddes DT, Kent J, et al. Infants of mothers with persistent nipple pain exert strong sucking vacuums. Acta Paediatr 2008;97:1205-1209.

14. Shamsa A, Bai J, Raviraj P, et al. Mode of delivery and its associated maternal and neonatal outcomes. Open J Obstet Gynaecol 2013;3:307-312.

15. Kearney MH, Cronenwett LR, Reinhardt R. Cesarean delivery and breastfeeding outcomes. Birth 1990;17:97-103.

16. Zanardo V, Pigozzo A, Wainer G, et al. Early lactation failure and formula adoption after elective caesarean delivery: Cohort study. Arch Dis Child Fetal Neonatal Ed 2013;98:F37F41.

17. Suzuki S, Hirohata S, Uriu K, et al. Cesarean delivery as a factor promoting exclusive breastfeeding in Japan. J Matern Fetal Neonatal Med 2013;26:1762-1763.

18. Wu M, Chason R, Wong M. Raynaud's phenomenon of the nipple. Obstet Gynecol 2012;119:447-449.

19. Anderson JE, Held N, Wright K. Raynaud's phenomenon of the nipple: A treatable cause of painful breastfeeding. Pediatrics 2004;113:e360-e364.

20. Lawlor-Smith L, Lawlor-Smith C. Vasospasm of the nipple-A manifestation of Raynaud's phenomenon: Case reports. BMJ 1997;314:644-645.

21. Page SM, McKenna DS. Vasospasm of the nipple presenting as painful lactation. Obstet Gynecol 2006;108:806-808.

22. Holmen OL, Backe B. An underdiagnosed cause of nipple pain presented on a camera phone. BMJ 2009;339:b2553.

23. McGuinness N, Cording V. Raynaud's phenomenon of the nipple associated with labetalol use. J Hum Lact 2013;29:17-19.

24. Lawlor-Smith CL, Lawlor-Smith LS. Raynaud's phenomenon of the nipple: A preventable cause of breastfeeding failure? Med J Aust 1997;166:448.

25. Amir LH, Cullinane M, Garland SM, et al. The role of microorganisms (Staphylococcus aureus and Candida albicans) in the pathogenesis of breast pain and infection in lactating women: Study protocol. BMC Pregnancy Childbirth 2011;11:54.

26. Amir LH, Donath SM, Garland SM, et al. Does Candida and/ or Staphylococcus play a role in nipple and breast pain in lactation? A cohort study in Melbourne, Australia. BMJ Open 2013;3:e002351.

27. Amir LH, James JP, Kelso G, et al. Accreditation of midwife lactation consultants to perform infant tongue-tie release. Int J Nurs Pract 2011;17:541-547.
28. Amir LH, Garland SM, Lumley J. A case-control study of mastitis: Nasal carriage of Staphylococcus aureus. BMC Fam Pract 2006;7:57.

29. Fitzmaurice GM. Longitudinal Data Analysis. Boca Raton, FL: Chapman \& Hall, 2009.

30. Newton N. Nipple pain and nipple damage: Problems in the management of breast feeding. J Pediatr 1952;41:411-423.

31. Doherty T, Sanders D, Jackson D, et al. Early cessation of breastfeeding amongst women in South Africa: An area needing urgent attention to improve child health. BMC Pediatr 2012;12:105.

32. Strong GD. Provider management and support for breastfeeding pain. J Obstet Gynecol Neonatal Nurs 2011;40:753764.

33. Mahmood I, Jamal M, Khan N. Effect of mother-infant early skin-to-skin contact on breastfeeding status: A randomized controlled trial. J Coll Physicians Surg Pak. 2011;21:601605.

34. Craig HJ, Dietsch E. 'Too scary to think about': First time mothers' perceptions of the usefulness of antenatal breastfeeding education. Women Birth 2010;23:160-165.

35. Shaikh U, Scott BJ. Extent, accuracy, and credibility of breastfeeding information on the internet. J Hum Lact 2005; 21:175-183.

36. Forster D, McLachlan H, Lumley J, et al. Two mid-pregnancy interventions to increase the initiation and duration of breastfeeding: A randomized controlled trial. Birth 2004; 31:176-182.

37. Colson SD, Meek JH, Hawdon JM. Optimal positions for the release of primitive neonatal reflexes stimulating breastfeeding. Early Hum Dev 2008;84:441-449.

38. Doucet $S$, Soussignan $R$, Sagot $P$, et al. An overlooked aspect of the human breast: Areolar glands in relation with breastfeeding pattern, neonatal weight gain, and the dynamics of lactation. Early Hum Dev 2012;88:119-128.

39. Goundry B, Bell L, Langtree M, et al. Diagnosis and management of Raynaud's phenomenon. BMJ 2012;344.

40. Bovenzi M, Franzinelli A, Strambi F. Prevalence of vibrationinduced white finger and assessment of vibration exposure among travertine workers in Italy. Int Arch Occup Environ Health 1988;61:25-34.

41. Fraenkel L. Raynaud's phenomenon: Epidemiology and risk factors. Curr Rheumatol Rep 2002;4:123-128.

42. Palmer KT, Griffin MJ, Syddall H, et al. Prevalence of Raynaud's phenomenon in Great Britain and its relation to hand transmitted vibration: A national postal survey. Occup Environ Med 2000;57:448-452.

43. Australian Institute of Health and Welfare. 2010 Australian National Infant Feeding Survey: Indicator Results. Canberra: Australian Institute of Health and Welfare, 2011.

44. Ahluwalia IB, Li R, Morrow B. Breastfeeding practices: Does method of delivery matter? Matern Child Health J 2012; 16:231-237.
Address correspondence to:

Miranda L Buck, BA, MPhil Mother and Child Health Research

La Trobe University

215 Franklin Street

Melbourne, VIC, 3000, Australia

E-mail: m.buck@latrobe.edu.au 